\title{
COMPACT HOLOMORPHICALLY CONVEX SUBSETS OF A STEIN MANIFOLD $\left({ }^{1}\right)$
}

\author{
BY \\ REESE HARVEY AND R. O. WELLS, JR.
}

1. Introduction. Let $X$ be a complex manifold which is countable at infinity and let $\mathcal{O}$ denote the sheaf of germs of holomorphic functions on $X$. Let $\mathcal{O}(U)$ be the Fréchet algebra of holomorphic functions on an open set $U \subset X$, with the topology of uniform convergence on compact subsets of $X$. Let $S$ be a subset of $X$, and define $\mathcal{O}(S)$ as the inductive limit

$$
\mathcal{O}(S)=\underset{U \supset S}{\operatorname{ind} \lim } \mathcal{O}(U)
$$

where $U$ runs over open subsets of $X$ which contain $S$, and suppose that $\mathcal{O}(S)$ has the inductive limit topology. Let $\tilde{S}$ be the spectrum $\left({ }^{2}\right)$ of the algebra $\mathcal{O}(S)$, and let $g: S \rightarrow \tilde{S}$ be the usual evaluation map defined by $g(x) f=f(x)$ for $f \in \mathcal{O}(S)$. We shall say that $S$ is holomorphically convex if $g$ is a bijective mapping onto $\tilde{S}$. If $S$ is an open subset of a Stein manifold $X$, then it is well known that $S$ is holomorphically convex if and only if $S$ is a Stein manifold (see [6]). If $S$ is compact we shall see (Proposition 2.4) that this definition of holomorphic convexity agrees with the definition given in [10] where various geometric characterizations are discussed.

The purpose of this paper is to prove Theorems A and B of Cartan for coherent analytic sheaves on a compact holomorphically convex subset of a Stein manifold $X$. We also give a characterization of holomorphic convexity of compact subsets of $X$ in terms of a weakened form of Theorem B. In §II we introduce a concept of the envelope of holomorphy of arbitrary subsets of a Stein manifold, generalizing the envelope of holomorphy of open subsets of a Stein manifold, whose existence was shown by Rossi [6]. In §III we prove the main theorems of the paper, using some of our results from §II.

II. Envelopes of holomorphy. Let $S$ be a subset of a Stein manifold $X$, and $\mathcal{O}(S)$ be as in $\S$ I. Let $\mathcal{O}^{\prime}(S)$, the dual of $\mathcal{O}(S)$ as a topological vector space, have the weak topology induced by $\mathcal{O}(S)$. This induces the weak topology on $\tilde{S} \subset \mathcal{O}^{\prime}(S)$. The restriction map from $\mathcal{O}(X)$ into $\mathcal{O}(S)$ has a continuous adjoint from $\mathcal{O}^{\prime}(S)$ into $\mathcal{O}^{\prime}(X)$ which obviously maps $\tilde{S}$ continuously into $\tilde{X}$. Since $X$ is Stein we can identify $X$ with $\tilde{X}$. This gives us a map from $\tilde{S}$ into $X$ which will be denoted by $\pi$. Let $g$ be the

Received by the editors August 14, 1967 and, in revised form, October 18, 1967.

(1) This research was supported by NSF Grants GP-7038 and GP-5951.

$\left.{ }^{2}\right) \tilde{S}$ is the set of nonzero continuous homomorphisms of the algebra $\mathcal{O}(S)$ into $C$. 
evaluation map from $S$ into $\tilde{S}$. We then have the following commutative diagram

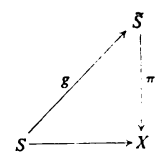

We shall need the following important result due to Rossi [6] in the case where $S=U$ is open in $X$ (see also [4, Chapter 1]).

THeOREM 2.1 (Rossi). Let $U$ be an open set in a Stein manifold $X$, then $U$ can be given the structure of a Stein manifold such that:

(a) $\pi$ is a locally biholomorphic map of $\tilde{U}$ into $X$.

(b) $g$ is an analytic isomorphism of $U$ into $\tilde{U}$.

(c) $g^{*}: \mathcal{O}(\tilde{U}) \rightarrow \mathcal{O}(U)$ is a Fréchet algebra isomorphism.

Now let us return to the case where $S$ is an arbitrary subset of the Stein manifold $X$. Suppose that $\left\{U_{\alpha}, \alpha \in I\right\}$ is a fundamental system of open neighborhoods of $S$, where $U_{\alpha} \subset U_{\beta}$ if and only if $\alpha>\beta$. If $\alpha>\beta$, then the restriction map $\mathcal{O}\left(U_{\beta}\right)$ into $\mathcal{O}\left(U_{\alpha}\right)$ has a continuous adjoint from $\mathcal{O}^{\prime}\left(U_{\alpha}\right)$ into $\mathcal{O}^{\prime}\left(U_{\beta}\right)$ which, when restricted to $\tilde{U}_{\alpha}$, defines a continuous map $\varphi_{\alpha \beta}$ from $\tilde{U}_{\alpha}$ into $\tilde{U}_{\beta}$. By Theorem 2.1(a), we see that $\varphi_{\alpha \beta}$ is the spreading map of $\tilde{U}_{\alpha}$ over $\tilde{U}_{\beta}$ obtained by considering $U_{\alpha}$ as an open subset of the Stein manifold $\tilde{U}_{\beta}$. This implies that $\varphi_{\alpha \beta}$ is locally biholomorphic.

In general, the weak dual of an inductive limit of locally convex spaces is the projective limit of the weak duals (see, for example, [8, p. 140]). Therefore

and hence

$$
\mathcal{O}^{\prime}(S)=\underset{\alpha \in I}{\operatorname{proj}} \lim \mathcal{O}^{\prime}\left(U_{\alpha}\right)
$$

$$
\tilde{S}=\operatorname{proj} \lim _{\alpha \in I} \tilde{U}_{\alpha}
$$

where the projective limits are taken with respect to the maps $\left\{\varphi_{\alpha \beta}\right\}$ defined above.

Let ${ }_{\alpha} \mathcal{O}$ denote the sheaf of germs of holomorphic functions on $\tilde{U}_{\alpha}$. These sheaves induce, in a natural way, a sheaf of rings on $\tilde{S}$. This sheaf will be called the holomorphic structure sheaf on $\tilde{S}$ and will be denoted by $\tilde{s}_{\tilde{O}} \mathcal{O}$. It is defined as follows. For each $U_{\alpha}$, the obvious map $\varphi_{\alpha}$ from $\tilde{S}$ into $\tilde{U}_{\alpha}$ induces an inverse image sheaf $\varphi_{\alpha}^{*}\left({ }_{\alpha} \mathcal{O}\right)$ on $\tilde{S}$, such that $\left(\varphi_{\alpha}^{*}\left({ }_{\alpha} \mathcal{O}\right)\right)_{x} \cong{ }_{\alpha} \mathcal{O}_{\varphi_{a}(x)}$. Also, the maps $\varphi_{\alpha \beta}$, from $\tilde{U}_{\alpha}$ into $\tilde{U}_{\beta}$ induce compatible sheaf homomorphisms

We then let

$$
\psi_{\alpha \beta}: \varphi_{\beta}^{*}\left({ }_{\beta} \mathcal{O}\right) \rightarrow \varphi_{\alpha}^{*}\left({ }_{\alpha} \mathcal{O}\right)
$$

$$
\tilde{s}^{\mathcal{O}}=\text { ind } \lim _{\alpha \in I} \varphi_{\alpha}^{*}\left({ }_{\alpha} \mathcal{O}\right)
$$

where the inductive limit is taken with respect to the maps $\left\{\psi_{\alpha \beta}\right\}$.

We have the following analogues of parts (a) and (b) of Theorem 2.1.

Proposition 2.2. (a) The map $\pi$ from $\tilde{S}$ into $X$ induces an algebraic isomorphism from $\mathcal{O}_{\pi(x)}$ onto $\tilde{S}_{\tilde{S}}$ for each $x \in \tilde{S}$. 
(b) The evaluation map $g$ is a homeomorphism from $S$ onto $g(S)$ which induces a sheaf isomorphism from $\left.{ }_{\tilde{S}} \mathcal{O}\right|_{g(S)}$ onto $\left.\mathcal{O}\right|_{S}$, if $S$ is identified with $g(S)$.

Proof. For each $x \in \tilde{S}, \tilde{S}_{\tilde{S}}$ is the inductive limit of the stalks $\left\{{ }_{\alpha} \mathcal{O}_{\varphi_{a}(x)}\right\}$. Since each $\varphi_{\alpha \beta}$ is a locally biholomorphic map, this implies that the map from ${ }_{\alpha} \mathcal{O}_{\varphi_{a}(x)}$ into $\mathcal{N}_{\bar{S}}$ is an isomorphism for each $\alpha \in I$. Now part (a) is just the special case where $U_{\alpha}=X$ and $\varphi_{\alpha}=\pi$. To prove part (b) first note that $g^{-1}$ is equal to $g_{\alpha}^{-1} \circ \varphi_{\alpha}$ and hence it is continuous. The remainder of part (b) follows from the fact that for each $\alpha \in I$, $\left.\left(\varphi_{\alpha}^{*}\left({ }_{\alpha} \mathcal{O}\right)\right)\right|_{g(S)}$ is isomorphic to $\left.\mathcal{O}\right|_{S}$ (under the identification of $S$ with $g(S)$ ), where this isomorphism is a consequence of part (b) of Theorem 2.1. Q.E.D.

It might happen that for some $S$, the set $\tilde{S}$ is embedded in a complex manifold in a natural way, then the stalk isomorphism in Proposition 2.2 implies that $\pi$ is "locally biholomorphic", by the inverse function theorem. The following proposition is an analogue of Theorem 2.1(c), and shows that holomorphic functions on $S$ can be "continued analytically" to the part of the spectrum connected to $S$.

Let $E(S)$ denote the components of $\tilde{S}$ which intersect $S$.

Proposition 2.3. The restriction map $g^{*}: \Gamma\left(E(S),{ }_{s} \mathcal{O}\right) \rightarrow \Gamma(S, \mathcal{O})$ is an algebraic isomorphism.

Proof. Suppose $f$ is a section of $\mathcal{O}$ over $S$. Then $f$ is the restriction of a section $f^{1}$ of $\mathcal{O}$ on some neighborhood $U_{\alpha}$ of $S$ (see [3]). By part (c) of Theorem 2.1, $f^{1}$ extends to a section $f^{2}$ of ${ }_{\alpha} \mathcal{O}$ on $\tilde{U}_{\alpha}$ (where we identify $U_{\alpha}$ with its image in $\tilde{U}_{\alpha}$ ). Then $f^{2}$ induces a section $f^{3}$ of $\tilde{s}_{\tilde{S}} \mathcal{O}$ over $\tilde{S}$, which when restricted to $S$ is just $f$ (again identifying $S$ with $g(S)$ ). This proves $g^{*}$ is onto. The fact that $g^{*}$ is one-to-one follows easily from the following principle of unique continuation for sections of $\tilde{s}_{\tilde{S}} \mathcal{O}$ and the assumption that $E(S)$ is connected to $S$. Suppose $\left\{x_{v}\right\}$ is a net in $\tilde{S}$ which converges to $x \in \tilde{S}$, and suppose that $f$ is a section of $\tilde{s}_{\tilde{S}} \mathcal{O}$ which is defined in a neighborhood of $\left\{x_{v}\right\} \cup x$, such that $f_{x_{v}}=0$ (as a germ) for all $\nu$, then $f_{x}=0\left(^{3}\right)$. To show this, let $h_{\varphi_{a}(x)}$ be the germ at $\varphi_{\alpha}(x)$ which induces the germ $f_{x}$ (see Proposition 2.2). The germ $h_{\varphi_{a}(x)}$ is induced by a section $h$ of ${ }_{\alpha} \mathcal{O}$ in some neighborhood of $\varphi_{\alpha}(x)$, and $h$ induces the section $f$ in some neighborhood of $x$. Therefore there exists a $\mu$ such that $h_{\varphi_{a}\left(x_{v}\right)}$ induces $f_{x}$ if $\nu>\mu$. This implies that $h_{\varphi_{\alpha}\left(x_{v}\right)}$ is the zero germ at $\varphi_{\alpha}\left(x_{v}\right)$ for $\nu>\mu$, since $_{\tilde{s}} \mathcal{O}_{x_{v}} \cong \mathcal{O}_{\varphi_{a}\left(x_{v}\right)}$ (see the proof of part (a) of Proposition 2.2). By the uniqueness of analytic continuation on $\tilde{U}_{\alpha}$ we obtain that $h_{\varphi_{a}(x)}$, and hence $f_{x}$, is the zero germ. Q.E.D.

Whether $E(S)$ is the same as $S$ or not is related to the following question. Let $A(S)$ denote the closure of $\mathcal{O}(S)$ in $C(S)$, where $C(S)$ is the space of continuous functions on $S$ with the topology of uniform convergence on compact subsets, and let $\mathscr{S}(A(S))$ be the spectrum of $A(S)$. Then there is a continuous map $\rho$ from $\mathscr{S}(A(S))$ into the set of $x \in \tilde{S}$ satisfying $|x(f)| \leqq \sup _{K}|f|$, for all $f \in \mathcal{O}(S)$ and for some compact set $K \subset S$. The Šilov idempotent theorem can be used to prove that

$\left(^{3}\right)$ If $f$ is a section of a sheaf, then $f_{x}$ denotes the germ of $f$ at $x$. 
$E(S)$ contains $\rho(\mathscr{S}(A(S))$ ) (see [4]). Therefore, if $\rho(\mathscr{S}(A(S)))$ is dense in $S$, then $E(S)=\tilde{S}$. If $S$ is open then trivially we have that $\rho(\mathscr{S}(A(S)))=\tilde{S}$.

Proposition 2.4. If $K$ is a compact set in a complex manifold $X$, then the spectra of the algebras $A(K)$ and $\mathcal{O}(K)$ are homeomorphic.

Remark. $\mathscr{S}(A(K))$ was called the holomorphic hull of $K$ in [10].

Proof. Suppose $x \in \tilde{K}$. We must show that

$$
|x(f)| \leqq \sup _{K}|f|, \quad \text { for all } f \in \mathcal{O}(K) .
$$

Suppose this were not so, then there would be a function $f \in \mathcal{O}(K)$ such that $x(f)=1$ but $\sup _{K}|f|<1$. These two facts imply that $x(1-f)=0$ and $(1-f)^{-1} \in \mathcal{O}(K)$. This is impossible, since then we would have

$$
1=x\left((1-f)(1-f)^{-1}\right)=x(1-f) x(1-f)^{-1}=0 .
$$

In analogy to Theorem 2.1(a) one could ask whether $\pi$ were "locally biholomorphic" in the sense of being a local homeomorphism which induces an isomorphism (locally) between $\mathcal{O}$ and ${ }_{\tilde{S}} \mathcal{O}$. The following example shows that this is not necessarily the case. Let

$$
\begin{aligned}
& B_{0}=\left\{(z, w) \in C^{2}:|z|^{2}+|w|^{2} \leqq 1\right\}, \\
& B_{n}=\left\{(z, w) \in C^{2}:|z|^{2}+|w|^{2} \leqq 1+1 / n\right\}, \quad n \geqq 1,
\end{aligned}
$$

and letting $\partial B_{n}$ be the boundary of $B_{n}$ we set

$$
S=\bigcup_{n=0}^{\infty} \partial B_{n}
$$

Then it is easy to see that set-theoretically (disjoint union)

$$
\widetilde{S}=\bigcup_{n=0}^{\infty} B_{n} .
$$

However $\tilde{S}$, which is equipped with the weak topology induced by $\mathcal{O}(S)$, is not mapped locally homeomorphically by $\pi$ into $C^{2}$. Suppose it were (which would be the case if $\tilde{S}$ were equipped with the disjoint union topology, for example), then, letting $p_{n}$ be the center of the ball $B_{n}$ in $\tilde{S},\left\{p_{n}\right\}$ would be a discrete set. But it follows easily from Hartogs' theorem that, in the weak topology on $\widetilde{S}$, the points $p_{n}$ converge to the point $p_{0}$, which is a contradiction. Thus $\tilde{S}$ is not a subset of a complex manifold in any natural way. The authors would like to thank the referee for supplying this example.

III. Compact holomorphically convex sets. In order to compute cohomology it will be convenient to express $\widetilde{K}$ as the projective limit of compact sets, in the case where $K$ is compact. Considering $K$ as a subset of $\tilde{U}_{\alpha}$, where we use the same notation as before, let $\widehat{K}_{\alpha}$ denote the $\mathcal{O}\left(\tilde{U}_{\alpha}\right)$-hull of $K$, i.e.,

$$
\hat{K}_{\alpha}=\left\{x \in \tilde{U}_{\alpha}:|f(x)| \leqq \sup _{K}|f|, \forall f \in_{\alpha} \mathcal{O}\left(\tilde{U}_{\alpha}\right)\right\}
$$


One can easily check that $\varphi_{\alpha}(\tilde{K}) \subset \hat{K}_{\alpha}$ and $\varphi_{\alpha \beta}\left(\hat{K}_{\alpha}\right) \subset \hat{K}_{\beta}$. Since $\tilde{U}_{\alpha}$ is a Stein manifold, it follows that $\hat{K}_{\alpha}$ is compact. Thus we have the important

LEMMA 3.1. If $K$ is compact in $X$, then $\tilde{K}$ is homeomorphic to the projective limit of the compact Hausdorff spaces $\hat{K}_{\alpha}$.

The projective limit of compact spaces retains one of the important properties of a fundamental neighborhood system.

Corollary 3.2. Suppose $N$ is a neighborhood of $\varphi_{\beta}(\tilde{K})$ in $\hat{K}_{\beta}$. Then there exists an $\alpha>\beta$ such that $\varphi_{\alpha \beta}\left(\hat{K}_{\alpha}\right) \subset N$.

Proof. Suppose this were not so. Then $\varphi_{\alpha \beta}^{-1}\left(\hat{K}_{\beta}-N\right)$ is a compact nonempty set for all $\alpha \geqq \beta$, which implies that there exists a point

$$
x \in \underset{\alpha}{\operatorname{proj} \lim } \varphi_{\alpha \beta}^{-1}\left(\hat{K}_{\beta}-N\right) \subset \underset{\alpha}{\operatorname{proj} \lim } \hat{K}_{\alpha}=\tilde{K}
$$

such that $\varphi_{\beta}(x) \notin N$. But this is a contradiction. Q.E.D.

We now have the following analogue to the Fundamental Theorem of Cartan for Stein manifolds (see [1], [2]).

THEOREM 3.3. Let $K$ be a compact holomorphically convex subset of a Stein manifold $X$, and let $\mathscr{F}$ be a coherent analytic sheaf on $K$. Then:

(A) For each $x \in K$ the $\mathcal{O}_{x}$-module $\mathscr{F}_{x}$ is generated by the germs at $x$ of $\Gamma(K, \mathscr{F})$.

(B) $H^{q}(K, \mathscr{F})=0$ for all $q>0$.

Proof. By Proposition 2 of Cartan [2] there exists a neighborhood $U$ of $K$ in $X$ and a coherent analytic sheaf $\mathscr{G}$ on $U$ which restricts to $\mathscr{F}$ on $K$. By Corollary 3.2 there exists a $\hat{K}_{\beta}$ such that $\pi_{\beta}\left(\hat{K}_{\beta}\right) \subset U$. The inverse image $\pi_{\beta}^{*} \mathscr{G}$ is a coherent analytic sheaf on a neighborhood of $\hat{K}_{\beta}$ in $\tilde{U}_{\beta}$ because $\pi_{\beta}$ is locally biholomorphic. Now, as is well known, $\hat{K}_{\beta}$ has a fundamental neighborhood system in $\tilde{U}_{\beta}$ consisting of Stein manifolds. In particular, there is an open Stein manifold $Y$ in $\widetilde{U}_{\beta}$ which contains $K$ such that $\pi_{\beta}^{*} \mathscr{G}$ is a coherent analytic sheaf on $Y$. Since $\pi_{\beta}^{*} \mathscr{G}$, when restricted to $K$, is just $\mathscr{F}$ (under the identification map $g_{\beta}: U_{\beta} \rightarrow \widetilde{U}_{\beta}$ ), we see that part (A) follows immediately from Cartan's Theorem A applied to $\pi_{\beta}^{*} \mathscr{G}$ on $Y$. Since, for each $\alpha, \hat{K}_{\alpha}$ has a fundamental neighborhood system consisting of Stein manifolds it follows as in [2] from Cartan's Theorem B that $H^{q}\left(\hat{K}_{\alpha}, \pi_{\alpha}^{*} \mathscr{G}\right)=0$, for all $q>0$ and all $\alpha>\beta$. Therefore, once we show that

$$
H^{q}(K, \mathscr{F})=\underset{\alpha \geqq \beta}{\text { ind } \lim } H^{q}\left(\hat{K}_{\alpha}, \pi_{\alpha}^{*} \mathscr{G}\right)
$$

part (B) will be proved.

To show $(*)$ we proceed as follows. Suppose $q$ is an integer $\geqq 0$. Let $\left\{\mathscr{C}^{\alpha, q}\right\}$ denote the canonical flabby resolution $\left(^{4}\right)$ of $\pi_{\alpha}^{*} \mathscr{G}$ restricted to $\hat{K}_{\alpha}$. Let $\left\{\mathscr{C}^{q}\right\}$ denote

() See, for example [3]. 
the canonical flabby resolution of $\mathscr{F}$ on $K$. Then there is a natural map

$$
\rho: \text { ind } \lim _{\alpha} \Gamma\left(\hat{K}_{\alpha}, \mathscr{C}^{\alpha, q}\right) \rightarrow \Gamma\left(K, \mathscr{C}^{q}\right) .
$$

We shall show that $\rho$ is an isomorphism. Suppose $f$ is a section of $\mathscr{C}^{q}$ on $K$, then (identifying $K$ with a subset of $\hat{K}_{\beta}$ ) it is easy to see that $f$ extends to a section of $\mathscr{C}^{\beta, q}$ on $\hat{K}_{\beta}$. Thus $\rho$ is onto. Suppose $f \in$ ind $\lim _{\alpha} \Gamma\left(\hat{K}_{\alpha}, \mathscr{C}^{\alpha, q}\right)$ is represented by $f^{\beta} \in \Gamma\left(\hat{K}_{\beta}, \mathscr{C}^{\beta, q}\right)$ and $f^{\beta}$ vanishes on $K$ (considered as a subset of $\hat{K}_{\beta}$ ). Then $f^{\beta}$ vanishes on a neighborhood $N$ of $K$ in $\hat{K}_{\beta}$. By Corollary 3.2 there exists an $\alpha \geqq \beta$ such that $\varphi_{\alpha \beta}\left(\hat{K}_{\alpha}\right) \subset N$. Therefore $f$ is represented in $\Gamma\left(\hat{K}_{\alpha}, \mathscr{C}^{\alpha, q}\right)$ by zero. This proves $\rho$ is one to one, and thus $(*)$ is established, since cohomology commutes with inductive limits. Q.E.D.

The following theorem gives a characterization of compact holomorphically convex sets, but first we need to make a definition. If $S \subset X$, then we shall say that $\mathscr{R}$ is a sheaf of relations on $S$ if $\mathscr{R}$ is the kernel of a sheaf homomorphism

$$
\left.\left.\mathcal{O}^{p}\right|_{S} \rightarrow \mathcal{C}^{q}\right|_{s}
$$

THeOrem 3.4. Suppose $K$ is a compact subset of a Stein manifold $X$. Then the following conditions are equivalent:

(a) $K$ is holomorphically convex.

(b) $H^{q}(K, \mathscr{F})=0$ for all $q>0$ and for all coherent analytic sheaves $\mathscr{F}$ on $K$.

(c) $H^{1}(K, \mathscr{R})=0$ for all sheaves of relations $\mathscr{R}$ on $K$.

Proof. The only thing we have to show is that (c) implies (a).

Assume (c) and suppose that $y \in \tilde{K}$. If there exists a point $x \in K$ such that $y(f)=0$ implies $f(x)=0$ for any $f \in \mathcal{O}(K)$, then $y=x$. This can be seen as follows: the function $f-y(f)$ satisfies $y(f-y(f))=0$, hence $f(x)-y(f)=0$, and by our identification we have $y=x$. Now assume that $y \in \tilde{K}$, but $y \notin K$. Then for each $x \in K$ there exists a function $f \in \mathcal{O}(K)$ such that $y(f)=0$, but $f(x) \neq 0$. By compactness of $K$, this implies that there exists functions $f_{1}, \ldots, f_{r} \in \mathcal{O}(K)$ such that $y\left(f_{j}\right)=0$, $j=1, \ldots, r$, and such that these functions do not vanish simultaneously at any point in $K$. This means that the map $\mu:\left.\left.\mathcal{O}^{r}\right|_{K} \rightarrow \mathcal{O}\right|_{K}$ defined by $\mu\left(g_{1}, \ldots, g_{r}\right)=\sum_{j} f_{j} g_{j}$ is surjective. We then define $\mathscr{R}$ by the exact sequence

$$
\left.\left.0 \rightarrow \mathscr{R} \rightarrow \mathcal{O O}^{r}\right|_{K} \stackrel{\mu}{\rightarrow} \mathcal{O}\right|_{K} \rightarrow 0 .
$$

By hypothesis, $H^{1}(H, \mathscr{R})=0$, and thus $\mu: \mathscr{C}(K)^{r} \rightarrow \mathcal{O}(K)$ is surjective. Hence there exists $g_{1}, \ldots, g_{r} \in \mathcal{O}(K)$ such that $\sum_{j} f_{j} g_{j}=1$. But this is impossible since $1=y(1)$ $=\sum_{j} y\left(f_{j}\right) y\left(g_{j}\right)=0$. Q.E.D.

As in the case of Stein manifolds, holomorphically convex sets will generally be topologically restricted. More precisely we have

THEOREM 3.5. Suppose $K$ is a compact holomorphically convex subset of a Stein manifold $X$, then $H^{q}(K, Z)=0$, for $q>\operatorname{dim}_{C} X$. 
Proof. If $Y$ is a Stein manifold of complex dimension $n$, then $H^{q}(Y, Z)=0$ for $q>n$ (see [11]). The theorem then follows from this fact in exactly the same way that (B) of Theorem 3.3 followed from Cartan's Theorem B. Q.E.D.

The following corollary generalizes the result that a Stein manifold of complex dimension $\geqq 2$ is connected at the boundary (see [4, p. 227]).

Corollary 3.6. Suppose $K$ is a compact holomorphically convex subset of a Stein manifold $X$ of complex dimension $n$, then

$$
H^{p}(X, Z) \cong H^{p}(X-K, Z), \quad p<n-1 .
$$

Proof. It follows from Alexander duality and Theorem 3.5 that $H_{K}^{p}(X, Z)=0$ for $p<n\left(H_{R}^{p}(X, Z)\right.$ is frequently denoted by $\left.H^{p}(X, X-K ; Z)\right)$. The corollary then follows from the exact sequence

$$
\cdots \rightarrow H_{K}^{p}(X, Z) \rightarrow H^{p}(X, Z) \rightarrow H^{p}(X-K, Z) \rightarrow \cdots \quad \text { Q.E.D. }
$$

REMARKS. (1) Suppose that $K$ is a holomorphically convex compact subset of an $n$-dimensional Stein manifold $X$ and $\mathscr{F}$ is a locally free analytic sheaf on $K$. By utilizing part (B) of Theorem 3.3 above one can show that (see [5] and [7]):

(i) $H_{K}^{q}(X, \mathscr{F})=0$ for $q \neq n$.

(ii) $H_{K}^{n}(X, \mathscr{F})=H^{0}\left(K, \mathscr{F} * \otimes \Omega^{n}\right)^{\prime}$.

(2) Suppose $K$ is a compact subset of a complex manifold $X$ with a fundamental system of Stein neighborhoods. Then $K$ is holomorphically convex (this follows immediately from (2.1) and the fact that $U=\tilde{U}$ if $U$ is Stein). It is unknown whether the converse is true.

(3) Suppose $M$ is a compact real $C^{\infty} k$-dimensional submanifold of an $n$ dimensional complex manifold $X$, and let $h_{x}=\operatorname{dim}_{C} T_{x}(M) \cap J T_{x}(M)$, the complex dimension of the holomorphic tangent space to $M$ at $x$. In this case we have a geometric characterization of the holomorphic convexity of $M$. Namely, under certain "generic" conditions on $M$, holomorphic convexity of $M$ is equivalent to $h_{x}$ being identically zero on $M$, and this in turn is equivalent to the apparently stronger assertion that $A(M)=C(M)$ (see [9] for a more complete discussion of these results).

\section{REFERENCES}

1. H. Cartan, "Variétés analytiques complexes et cohomologie," pp. 41-55, in Colloque sur les fonctions de plusiers variables, Brussels, 1953.

2. - Variétés analytiques réelles et variétés analytiques complexes, Bull. Soc. Math. France 85 (1957), 77-99.

3. R. Godement, Topologie algébrique et théorie de faisceaux, Hermann, Paris, 1958.

4. R. C. Gunning and H. Rossi, Analytic functions of several complex variables, Prentice-Hall, Englewood Cliffs, N. J., 1965.

5. A. Martineau, Les hyperfunctions de M. Sato, Séminaire Bourbaki, 13 (1961).

6. H. Rossi, On envelopes of holomorphy, Comm. Pure Appl. Math. 16 (1963), 9-17.

7. M. Sato, Theory of hyperfunctions. II, J. Fac. Sci. Univ. Tokyo Sect. I 8 (1960), 387-437. 
8. H. H. Schaefer, Topological vector spaces, Macmillan, New York, 1966.

9. R. O. Wells, Jr., "Holomorphic hulls and holomorphic convexity," in Proceedings of the conference on complex analysis, Rice University, 1967, Rice Univ. Studies, Vol. 54, Houston, Texas, (to appear).

10. - Holomorphic hulls and holomorphic convexity of differentiable submanifolds, Trans. Amer. Math. Soc. 132 (1968), 245-262.

11. A. Andreotti and T. Frankel, The Lefschetz theorem on hyperplane sections, Ann. of Math. 69 (1959), 713-717.

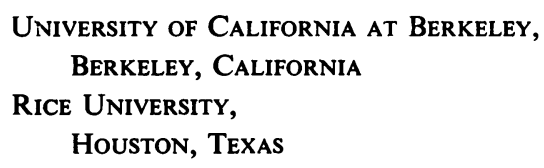

